\title{
A novel combined fluorescent probe staining method for circulating tumor cell identification
}

\author{
Huansheng Wang ${ }^{1,2 \#}$, Fajun Pei ${ }^{2 \#}, \mathrm{Hao} \mathrm{Li}^{3}$, Bing $\mathrm{Li}^{3}$, Shili Han ${ }^{4}$, Huajie $\mathrm{Yu}^{5}$, Sheng $\mathrm{Li}^{1,6}$ \\ ${ }^{1}$ Cheeloo College of Medicine, Shandong University, Jinan, China; ${ }^{2}$ Shandong Cancer Hospital and Institute, Shandong First Medical University and \\ Shandong Academy of Medical Sciences, Jinan, China; ${ }^{3}$ Shandong Pharmaceutical Research Institute, Jinan, China; ${ }^{4}$ Wuhan YZY Medical Science \\ and Technology Co., Wuhan, China; ${ }^{5}$ Department of Anesthesiology, Zhangqiu District People's Hospital, Jinan, China; ${ }^{6}$ Shandong First Medical \\ University, Jinan, China \\ Contributions: (I) Conception and design: S Li; (II) Administrative support: S Li; (III) Provision of study materials or patients: H Wang, F Pei, H \\ Li, B Li, S Han, H Yu; (IV) Collection and assembly of data: H Wang, S Han, H Yu; (V) Data analysis and interpretation: H Wang, S Han, H Yu; \\ (VI) Manuscript writing: All authors; (VII) Final approval of manuscript: All authors. \\ "These authors contributed equally to this work. \\ Correspondence to: Sheng Li. Cheeloo College of Medicine, Shandong University, Jinan, China. Email: drlisheng@sohu.com; Huajie Yu. Department \\ of Anesthesiology, Zhangqiu District People’s Hospital, Jinan 250000, China. Email: yuhj0414@163.com.
}

Background: To develop a novel highly accurate circulating tumor cell (CTC) identification method and to validate its application in cancer diagnostics and/or prognostics.

Methods: We verified and validated the combined fluorescent probe staining protocol (combination of three fluorescent probes: Dil, Hoechst 33342, and PY) through CTC and non-CTC (white blood cell) morphological comparison of five tumor cell lines (THP-1, HEC, HEPG2, Eca-109, HeLa) in vitro and 32 patient tumor samples from the Shandong Cancer Hospital and Institute. Wright's Giemsa staining and cluster differentiation 45 (CD45) immunocytochemistry (ICC) staining were used as reference control methods. The association between the developed method and clinicopathology was also investigated.

Results: We successfully developed and optimized the protocol, and validated the use of combined fluorescent probe staining for the identification of CTCs in the peripheral blood (PB) of tumor cell lines and tumor patients. Comparable CTC and non-CTC morphologies were observed for combined fluorescent probe staining and Giemsa staining methods in vitro. However, in vivo comparison between the three staining methods revealed that the identified CTCs differed in cell diameter and nucleo-cytoplasmic ratio. In addition, a higher CTC detection rate of 14/32, lower standard deviation (SD), and higher area under the receiver operating characteristic (ROC) curve (AUC) value of 0.844 were noted for combined fluorescence staining. Clinicopathological analysis revealed that CTCs were correlated with platelet levels $(\mathrm{P}=0.031)$, but not with age, gender, drinking history, or granule ratio.

Conclusions: We developed a combined fluorescent probe staining method with higher CTC identification accuracy than Wright's Giemsa staining, and propose this technique as a novel clinical diagnostic/prognostic tool.

Keywords: Fluorescent probe; circulating tumor cell (CTC); immunocytochemistry (ICC); identification

Submitted Oct 27, 2021. Accepted for publication Jan 05, 2022.

doi: 10.21037/atm-21-6476

View this article at: https://dx.doi.org/10.21037/atm-21-6476 


\section{Introduction}

Increased rates of morbidity and mortality have made cancer the leading cause of death in China (1), with more than $90 \%$ of cancer deaths being due to tumor metastasis (2). Pathological diagnosis has been the traditional method of cancer diagnosis and prognosis. In 1889, the British pathologist, Paget (3), proposed that circulating tumor cells (CTCs) act as "seeds" in tumorigenesis and metastasis. CTCs refer to tumor cells that enter the peripheral blood (PB) circulatory system from the primary tumor or metastatic lesions either spontaneously or due to diagnosis and treatment $(4,5)$. Only a few tumor cells are metastatic. CTCs are the key cells of cancer metastasis. CTCs can serve as biomarkers to assist in the physician's assessment of the likelihood of disease recurrence and survival prognosis of a patient (6-9). Monitoring the change trend of CTC type and quantity contributes to real-time individual treatment.

CTC identification is a complicated multi-step process involving cell separation, enrichment, and detection. The challenge of CTC detection and identification is primarily due to the low quantity of CTCs in PB relative to the high numbers of blood cells and platelets (10). Because the content of CTC in blood is very low, the detection is relatively difficult, and different detection methods have their own advantages and disadvantages. At present, there is no "gold standard" method for CTC identification (11-14). The only commercially available CTC identification technology is the FDA-approved CellSearch ${ }^{\circledR}$ system (Menarini Silicon Biosystems, Huntingdon Valley, PA, USA), which is coupled with proprietary immunomagnetic detection technology. There are many studies about the fluorescent probe staining method for CTC identification. However, the primary issue with the current CTC identification methods is the lack of a fully automated classification system that supports accurate consensus comparison across different medical establishments. Thus, CTC detection and assignment is subjective. The key challenge of CTC detection is establishing the consensus criteria for CTC assignment to increase the false positive rejection rate and successfully separate trapped CTCs (15-17). In previous studies, we developed the isolation by size of epithelial tumor cellsimmunocytochemistry (ISET-ICC) detection system, which identifies CTCs via physical isolation and enrichment, followed by subsequent morphological identification using the "negative exclusion" method (18-22). To further improve and simplify CTC identification following ISET isolation, we explored a novel direct CTC identification method in the present study using a staining method comprising the combination of three fluorescent probes: Dil, Hoechst 33342, and PY.

To validate the feasibility of identifying CTCs using the combined fluorescent probe staining method, we compared its performance against Wright's Giemsa staining and cluster differentiation 45 (CD45) ICC staining methods. We selected five tumor cell lines (HEC, EC109, THP-1, HEPG2, and HeLa) and recruited 32 patients with malignant tumors of various cancers who were treated at the Shandong Cancer Hospital and Institute (China) to establish the combined fluorescent probe staining method protocol as well as the CTC identification criteria. We assessed the accuracy of combined fluorescent probe staining relative to Wright's Giemsa staining in CTC identification by calculating the area under the receiver operating characteristic (ROC) curve (AUC). In addition, we investigated the relationship between CTC identification by combined fluorescent probes and cancer clinicopathology.

We present the following article in accordance with the MDAR reporting checklist (available at https://atm. amegroups.com/article/view/10.21037/atm-21-6476/rc).

\section{Methods}

\section{Cell lines and culture}

Five tumor cell lines (cute monocytic leukemia THP-1, human endometrial adenocarcinoma HEC, HEPG2, esophageal carcinoma Eca-109, and cervical cancer HeLa) were used in the present study. The HeLa cell line (at the logarithmic growth phase) was provided by Professor Yu Xiaoqiang from the Functional Crystal Materials Laboratory of Shandong University, China. The remaining four cell lines (THP-1, HEC, HEPG2, and Eca-109) were a gift from Wang Xingwu from the Central Laboratory of Shandong Cancer Hospital, China. Single cell suspensions were prepared by $0.25 \%$ trypsin digestion at $37^{\circ} \mathrm{C}$.

\section{Patient information and grouping}

Thirty-two patients with malignant tumors admitted into Shandong Cancer Hospital and Institute (China) from May, 2017 to September, 2017 were enrolled in the present study based on the inclusion and exclusion criteria. The inclusion criteria were as follows: (I) patients with a clear clinical or pathological diagnosis; (II) patients aged $\geq 18$ years old; and (III) signed consent from both patients and their families. 
The exclusion criteria were as follows: (I) patients with a secondary malignant tumor; (II) patients with a history of skin diseases; or (III) those with severe vascular diseases, such as vasculitis. All procedures performed in this study involving human participants were in accordance with the Declaration of Helsinki (as revised in 2013). The necessary approval was obtained from the Ethics Committee of Shandong Cancer Hospital and Institute, China (No. 201702019). Written consent was obtained from all eligible subjects before enrollment into the study. The inclusion criteria for patient selection and recruitment were decided based on the results of the in vitro comparison between the combined fluorescence staining and Wright's Giemsa staining methods.

\section{Combined fluorescence staining}

Combined fluorescent probe staining was performed after ISET isolation of CTCs. The mixture of three fluorescent probes [Hoechst 33342 (Molecular Probes, molecular probe is a technology based on molecular hybridization, which uses probes to detect nucleic acid sequences with complementary sequences), PY (provided by Professor Yu Xiaoqiang), and Dil (Molecular Probes, molecular probe is a technology based on molecular hybridization, which uses probes to detect nucleic acid sequences with complementary sequences); $5 \mu \mathrm{M}$ each] were diluted with phosphate-buffered saline (PBS). The coverslip was incubated with $100 \mu \mathrm{L}$ of probe mixture at room temperature for $30 \mathrm{~min}$ before the solution was removed and the coverslip was washed three times with PBS. Cell imaging was performed using a fluorescence microscope. Three channels were used for cell imaging to obtain a tri-colored fluorescent image: first channel for Hoechst 33342 at $405 \mathrm{~nm}$ excitation and 420-470 nm emission wavelengths; second channel for PY at $405 \mathrm{~nm}$ excitation and 500-560 $\mathrm{nm}$ emission wavelengths; and the third channel for Dil at $543 \mathrm{~nm}$ excitation wavelength and 560-600 nm emission wavelengths. The nuclei were stained blue (first channel), nucleoli green (second channel), and cell membrane red (third channel). We determine the dose of fluorescent probe according to previous studies (18-22). Three random fields were selected for each slide, and three intact cells were randomly selected in each field for separate measurement. ImageJ (National Institutes of Health, Maryland, USA) was used for image analysis.

\section{Wright's Giemsa staining}

The same samples that were subjected to combined fluorescent probe staining were subsequently subjected to Giemsa staining. The filter was soaked with $300 \mu \mathrm{L}$ of Diff-A stain for 1 min before dilution with $100 \mu \mathrm{L}$ of PBS. Excess stain was blotted and removed before staining for 2 min with Diff-B solution (about $300 \mu \mathrm{L}$ ), which was also diluted with PBS before removal. The filter was then rinsed with distilled water. Diff-B residue on the filter was cleared to ensure that the color of Diff-B remains on the filter. The stained filter was then transferred to a slide and dried for $30 \mathrm{~min}$ in a $50{ }^{\circ} \mathrm{C}$ dry box. Sealing of the coverslip over the slide was then performed by drying for $30 \mathrm{~min}$. Glycerin was then added dropwise before visualization under an optical microscope. Three random fields were selected for each slide, and three intact cells were randomly selected in each field for separate measurement. ImageJ (National Institutes of Health, Bethesda, MD, USA) was used for image analysis.

\section{CTC assignment criteria}

The morphological characteristics of CTCs, including cell diameter, nuclear diameter, surface area, and nucleocytoplasmic ratio, were used for assessment. The morphological criteria for CTC assignment for both combined fluorescence staining and Giemsa staining are as follows: (I) large variation in cell size (ratio $>0.5$ ); (II) large nuclear diameter $>24 \mu \mathrm{m}$; (III) irregular shape of nucleus; (IV) >3 large nucleoli; and (V) high nuclei quality ratio. A cell is considered a CTC if it fulfills at least four of the above criteria. The consistency of the assessed morphological characteristics between the two staining methods was calculated as part of the verification of the protocol for combined fluorescent probe staining.

\section{CD45 ICC staining}

The same samples that were subjected to Giemsa staining were subsequently subjected to CD45 ICC staining. Glycerin-sealed slides with coverslips were rinsed with distilled water before being immersed in $100 \%$ ethanol for $1 \mathrm{~min}$, followed by $95 \%$ ethanol for $1 \mathrm{~min}$, and finally $75 \%$ ethanol for $20 \mathrm{~min}$. After ensuring sufficient removal of the dye, the sample slide was immersed in a dye bath containing distilled water and rinsed for $5 \mathrm{~min}$. Triton $\mathrm{X}-100(100 \mu \mathrm{L} ; 0.1 \%)$ was added dropwise to the slide and was incubated for $15 \mathrm{~min}$ at room temperature before washing for $2 \mathrm{~min} \times 3$ times with distilled water. Hydrogen peroxide $(100 \mu \mathrm{L} ; 0.3 \%)$ was added dropwise and incubated 
for $10 \mathrm{~min}$ at room temperature to perforate the cell membrane before washing for $2 \mathrm{~min} \times$ four times with PBS. CD45 primary antibody $(100 \mu \mathrm{L})$ was added dropwise and incubated for $1 \mathrm{~h}$ at room temperature to block endogenous peroxidase before washing for $2 \mathrm{~min} \times$ four times with PBS. Diaminobenzidine (DAB) dye $(100 \mu \mathrm{L})$ was added to the sample simultaneously with the primary antibody and was incubated at room temperature till visualization of color development under the microscope. The DAB dye was removed upon completion of color development and was rinsed with running water for $5 \mathrm{~min}$ before hematoxylin staining for $5 \mathrm{~min}$. The sample slide was then incubated with $100 \mu \mathrm{L}$ of horseradish peroxidase (HRP)-conjugated goat anti-rabbit/mouse secondary immunoglobulin G (IgG) for $15 \mathrm{~min}$ at room temperature before rinsing with PBS for $2 \mathrm{~min} \times$ four times. For nuclear staining, the sample was dehydrated with hydrochloric acid for 8 seconds, rinsed with distilled water for $5 \mathrm{~min}$, and then subjected to gradient alcohol dehydration (75\% ethanol for $1 \mathrm{~min}$, $95 \%$ ethanol for $1 \mathrm{~min}, 100 \%$ ethanol for $1 \mathrm{~min}$ ), air-drying, and finally resin sealing. Cell imaging was performed using a light microscope.

The current standard CD45 ICC morphological criteria for CTC identification are as follows: (I) large variation in size of nucleus relative to size of cell (ratio $>0.5$ ); (II) large nuclear diameter $>24 \mu \mathrm{m}$; (III) irregular shape of nucleus; (IV) three-dimensional chromatin staining; and (V) high nucleo-cytoplasmic ratio. A cell is assigned as a CTC if it fulfills at least four of the above criteria.

\section{Statistical analysis}

Statistical analysis was performed using the SPSS v2.0 software (IBM, Chicago, IL, USA). The paired $t$-test was used for morphological comparison of the cell diameter, nuclear diameter, cell surface area, and nucleo-cytoplasmic ratio between the three staining methods. The Chisquared test was performed for comparisons between the three paired datasets. $\mathrm{P}<0.5$ was considered not statistically significant.

\section{Results}

In vitro confirmation of CTC identification protocol via morphological comparison between combined fluorescent probe staining and Giemsa staining in five cell lines

The SOP of the proposed novel combined fluorescent probe staining method was validated by comparing with cells subjected to Wright's Giemsa staining. Five tumor cell lines were respectively stained with either combined fluorescent probes or Giemsa stain, and their morphological characteristics were compared.

As we intended to use the THP-1 and HEC cell lines for preliminary tests to aid in the execution of downstream experiments, we only collected one data set for the combined fluorescent probe staining of THP-1 cells and one set for the Wright's Giemsa staining of HEC cells (Tables 1,2). Thus, comparison within groups could not be performed. We found no statistically significant differences in the mean values of cell diameter, nuclear diameter, cell surface area, and nucleo-cytoplasmic ratio of the HEPG2 (Table 3, Figures 1,2), Eca-109 (Table 4), and HeLa (Table 5) cell lines between the Wright's Giemsa staining and combined fluorescent probe staining methods.

Comparison of the mean values of the cell morphological characteristics examined for the two groups of five tumor cell lines subjected to either Wright's Giemsa staining or combined fluorescent probe staining showed consistent morphological characteristics (Table 6). These results demonstrate the feasibility of using the proposed novel combined fluorescent probe staining method on tumor cell lines. The morphological characteristics (cell diameter, nuclear diameter, surface area and nucleo-cytoplasmic ratio) of CTC ( $\mathrm{P}=0.826,0.901,0.560$, and 0.750) and white blood cells $(\mathrm{P}=0.157,0.466,0.446$, and 0.475$)$ were consistent for both staining methods.

\section{Optimization of combined fuorescent probe staining protocol for CTC and non-CTC identification}

The inclusion criteria used for patient recruitment were based on the results of the comparison between the combined fluorescent probe staining and Giemsa staining methods. Patients who were excluded from this study had malignant tumors with a secondary malignancy (five cases), a history of dermatosis, suffered from severe vascular disease, such as vasculitis (four cases of esophageal cancer and one case of liver cancer), or had undergone a clinical validation experiment that used combined fluorescent probe staining or Wright's Giemsa staining.

For optimization of the combined fluorescent probe staining protocol for CTC identification, we used the selection (inclusion and exclusion) criteria as a reference, coupled with the PB samples of five tumor patients. Wright's Giemsa staining and CD45 ICC staining were 
Table 1 Comparison of the morphological characteristics of THP-1 acute monocytic leukemia cells subjected to either combined fluorescent probe staining or Wright's Giemsa staining

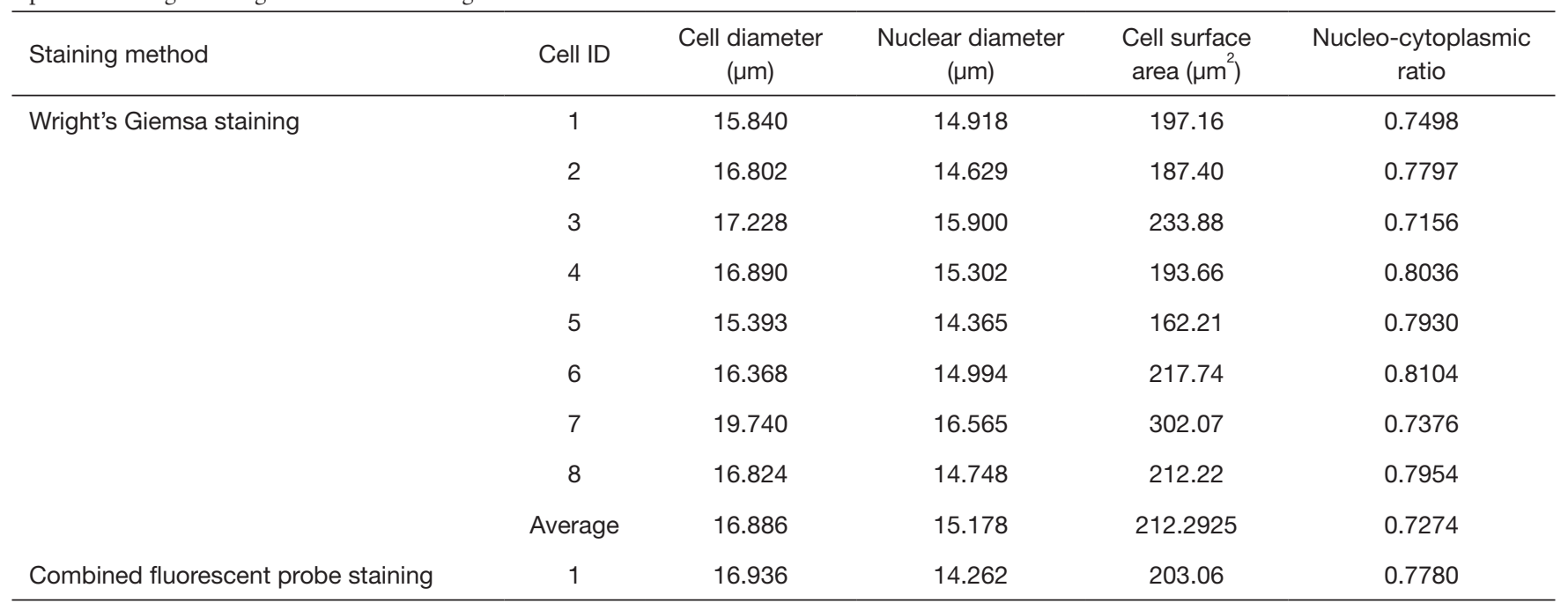

ID, identification.

Table 2 Comparison of the morphological characteristics of HEC endometrial adenocarcinoma cells subjected to either combined fluorescent probe staining or Wright's Giemsa staining

\begin{tabular}{lccccc}
\hline Staining method & Cell ID & $\begin{array}{c}\text { Cell diameter } \\
(\mu \mathrm{m})\end{array}$ & $\begin{array}{c}\text { Nuclear diameter } \\
(\mu \mathrm{m})\end{array}$ & $\begin{array}{c}\text { Cell surface } \\
\text { area }^{2}\left(\mu \mathrm{m}^{2}\right)\end{array}$ & $\begin{array}{c}\text { Nucleo-cytoplasmic } \\
\text { ratio }\end{array}$ \\
\hline Wright's Giemsa staining & 1 & 11.774 & 9.148 & 118.240 & 0.5828 \\
& 2 & 12.074 & 9.844 & 9.5215 & 110.717 \\
& 3 & 10.740 & 9.197 & 100.128 & 109.6950 \\
Combined fluorescent probe staining & Average & 11.5293 & 9.3963 & 10.515 & 102.915 \\
\hline
\end{tabular}

ID, identification.

used as experimental controls for finalization of the combined fluorescent probe staining method. The cell morphologies of both CTCs and non-CTCs (white blood cells) were assessed in terms of cell diameter, nuclear diameter, cell surface area, and nucleo-cytoplasmic ratio. We measured all CTCs, including cells that were suspected to be CTCs. Three random fields and three cells (CTCs or non-CTCs) from each field (total of nine cells) for each sample were selected and measured separately, followed by Image J analysis.

Comparison of combined fluorescent probe staining $v s$. Giemsa staining or CTC identification in the PB of five tumor patients (YG01 to YG05; one case of liver cancer and four cases of esophageal cancer) revealed that $2 / 5$ cases (YG04 and YG05) were detected by combined fluorescent probe staining, while no cases were identified by both Giemsa staining and CD45 ICC staining (Table 7). Thus, the relatively higher CTC detection rate of the developed combined fluorescent probe staining method verified the protocol and selection criteria used for clinical identification of CTCs in the PB of tumor patients.

To assess the non-CTC (white blood cell) detection rate of the combined fluorescent probe staining method, only the white blood cells in the PB of patient YG04 Li $\times$ Cheng were photographed and measured. Comparisons between the three staining methods were made by comparing between pairs (pair 1: Giemsa staining-combined fluorescent probe staining; pair 2: combined fluorescent probe staining-CD45 ICC staining; pair 3: Wright's Giemsa staining-CD45 ICC staining). No significant differences 
Table 3 Comparison of the morphological characteristics of HEPG2 cancer cells subjected to either combined fluorescent probe staining or Wright's Giemsa staining

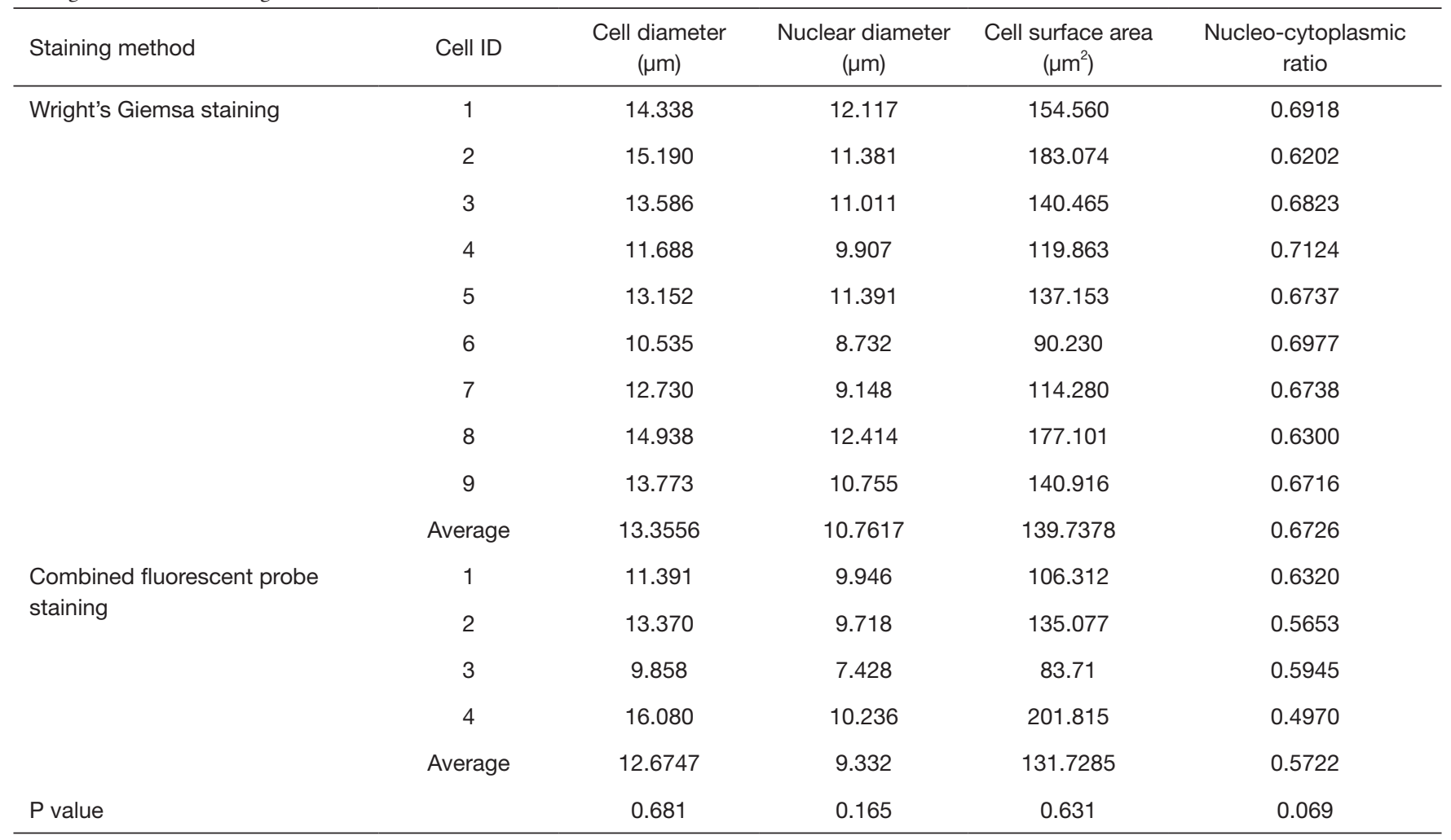

ID, identification.

in morphological characteristics were observed among the three staining methods (Table 8).

\section{Clinical validation of the combined fuorescent probe CTC identification method with 32 patients}

We confirmed the feasibility of the combined fluorescent probe staining method and finalizing the protocol in both in vitro (five tumor cell lines) and in vivo (five cancer patients) samples, and also confirmed the cell morphological consistency with both Wright's Giemsa staining and CD45 ICC staining methods. Next, we validated and assessed the performance of the developed combined fluorescent probe staining method in 32 cases of malignant tumor patients that were admitted into Shandong Cancer Hospital and Institute from May, 2017 to September, 2017 (including liver cancer, esophageal cancer, prostate cancer, kidney cancer, bladder cancer, and lung cancer; Table 8).

We had initially recruited 49 tumor patients for the present study and collected the PB samples from these patients. The patients were given a patient identity (ID) tag

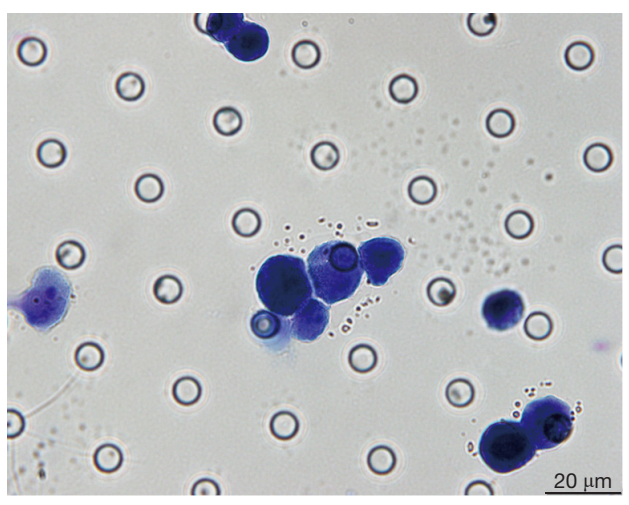

Figure 1 HEPG2 cell line (Giemsa staining; ×40).

from YG01-YG49 based on the chronological order of PB collection. The samples of patients YG01-YG30, YG34, and YG42 were used for the subsequent performance assessment of CTC and non-CTC identification compared to Wright's Giemsa staining and CD45 ICC staining. Staining by all three methods was not performed for the PB samples of 17 patients YG31-YG33, YG35-YG41, and 

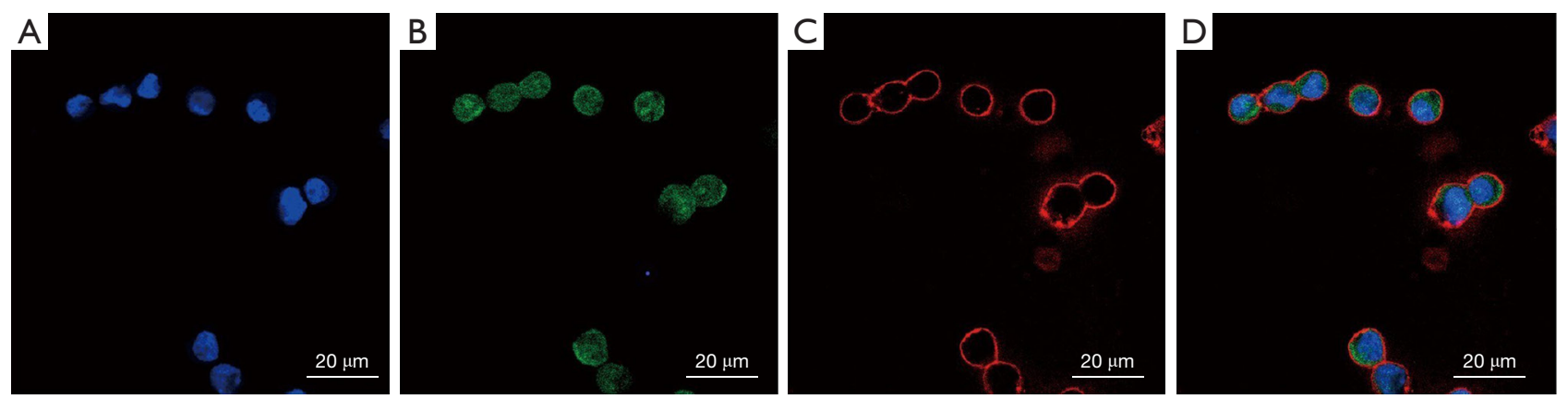

Figure 2 HEPG2 cell line [fluorescent probe; (A) blue marks the nucleu; (B) green marks the cytoplasmic; (C) red marks the cell membrane; (D) red and blue mark the fluorescent combination $\times 40]$.

Table 4 Comparison of the morphological characteristics of Eca-109 esophageal carcinoma cells subjected to either combined fluorescent probe staining or Wright's Giemsa staining

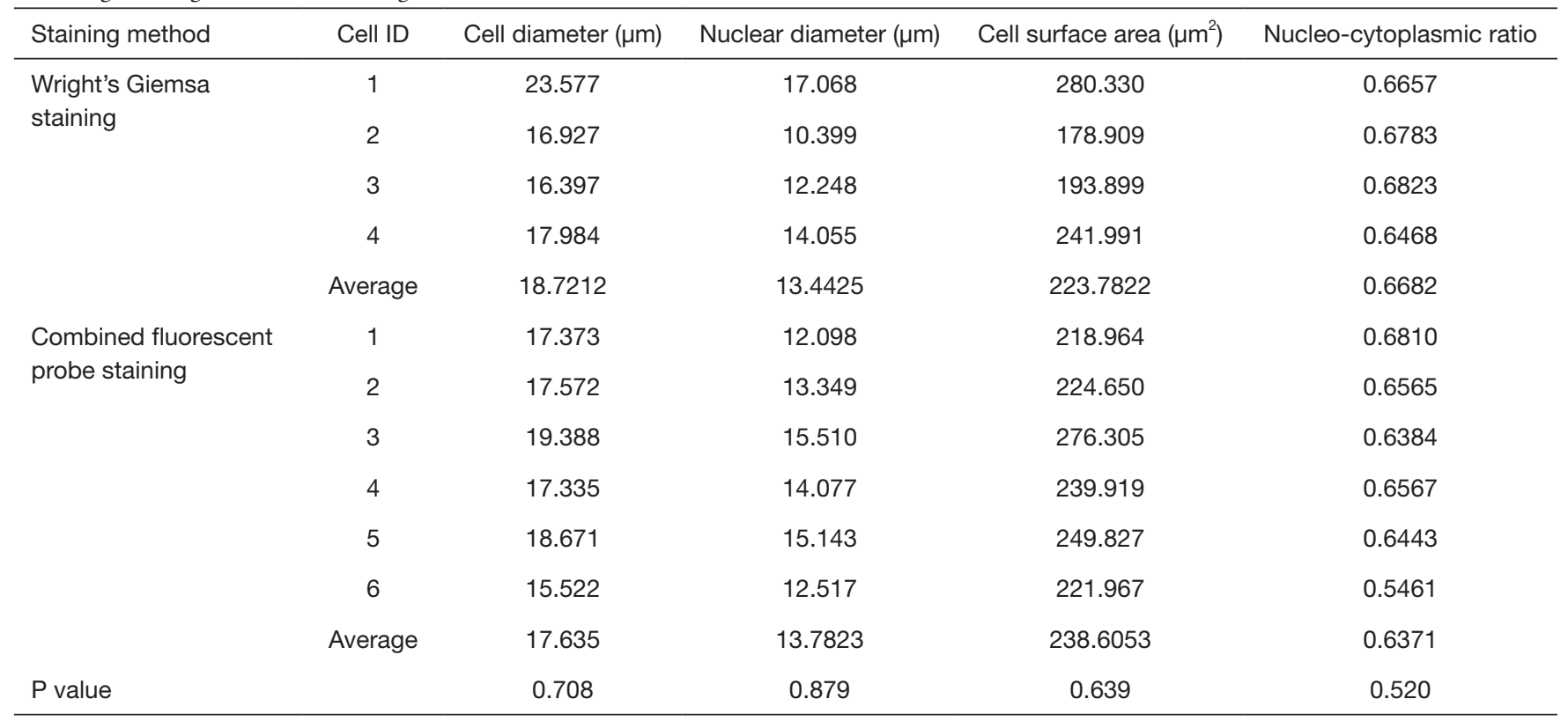

ID, identification.

YG43-YG49 (Figures 3-5). The CTC detection rates in the $\mathrm{PB}$ of the 32 tumor samples for the three staining methods were as follows: $16 / 32$ for combined fluorescent probe staining; 8/32 for Wright's Giemsa staining; and 6/32 for CD45 ICC staining.

\section{Comparison of non-CTC clinical detection accuracy between the three staining methods}

The lower standard deviation (SD) of each morphological characteristic for the combined fluorescence staining method compared to the other two staining methods (cell diameter: 1.01652 vs. 1.16724 and $1.12383 \mu \mathrm{m}$ for Giemsa and CD45 ICC staining respectively; nuclear diameter: 0.34397 vs. 0.64424 and $0.62349 \mu \mathrm{m}$; cell surface area: 12.79116 vs. 13.37422 ; and $13.13872 \mu^{2}$; nucleocytoplasmic ratio: 0.06341 vs. 0.07474 and 0.07231 ) indicated a higher accuracy for the developed combined fluorescence staining method (Tables 9-11). The degree of skewness between 0.05 and 0.35 signified that the data was statistically significant.

The non-CTCs identified and selected for the combined fluorescence staining method were as follows: three cells in YG03; two cells in YG04; one cell in YG08; and one cell 
Table 5 Comparison of cell the morphology characteristics of HeLa cervical cancer cells subjected to either combined fluorescent probe staining or Wright's Giemsa staining

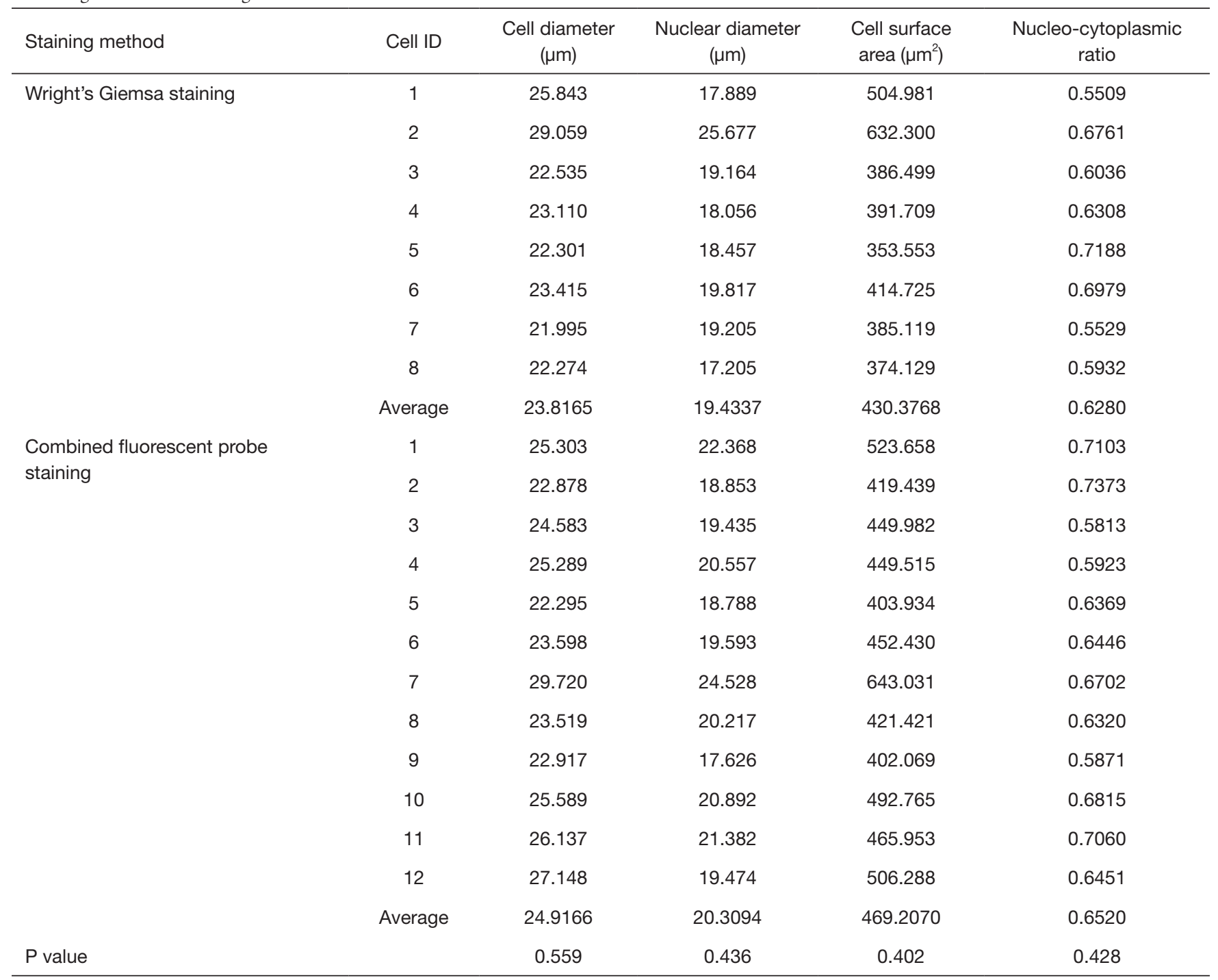

ID, identification.

in YG09. Eight cells in YG04 were identified for Wright's Giemsa staining method and five cells in YG04 were identified for CD45 ICC staining method. Comparison of the morphological parameters (cell diameter, nuclear diameter, cell surface area and nucleo-cytoplasmic ratio) of non-CTCs revealed no significant difference in any parameter among the three staining methods (Table 12).

\section{Comparison of CTC clinical detection accuracy between combined fluorescent probe staining and Giemsa staining}

The morphological characteristics of CTCs in the PB of
32 tumor samples were assessed and compared between combined fluorescent probe staining and Giemsa staining. The CD45 ICC staining method was not included in the comparison of the CTC morphological parameters. The lower SD of each morphological characteristic for the combined fluorescent probe staining method compared to Giemsa staining indicated a higher accuracy for the developed combined fluorescence staining method (Tables 13,14). The degree of skewness between 0.05 and 0.35 signified that the data was statistically significant. Comparison of the morphological parameters between the two staining methods revealed no significant differences 
Table 6 Comparison of the cell morphological characteristics between the five cell lines for the two staining methods, Wright's Giemsa staining vs. combined fluorescent probe staining, respectively

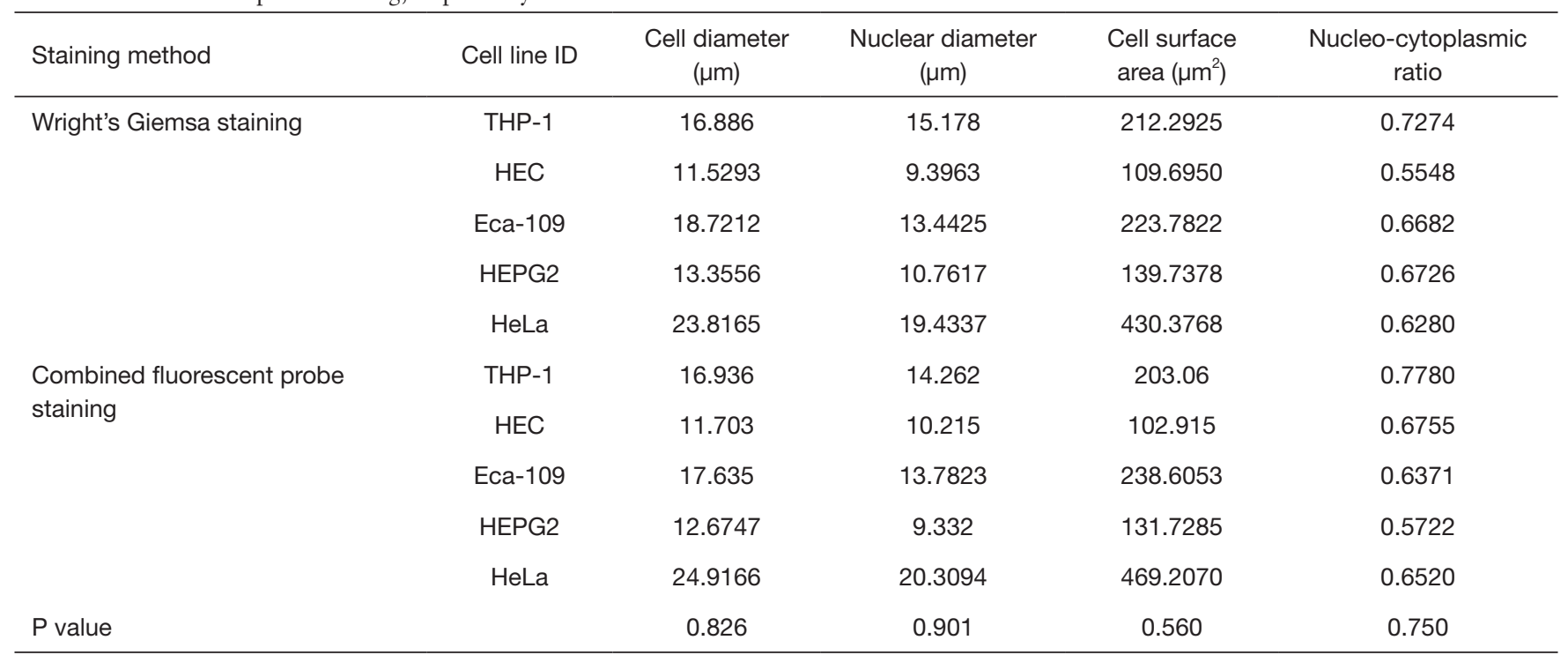

ID, identification.

in cell diameter and cell surface area $(\mathrm{P}=0.308$ and 0.147$)$; however, significant differences in nuclear diameter and nucleo-cytoplasmic ratio ( $\mathrm{P}=0.013$ and 0.004; Table 15) were observed. Notably, the difference was larger for the combined fluorescent probe staining method.

In a previous study, we established the ISET-ICC (ISET + CD45 ICC staining) method for the identification of CTCs in $\mathrm{PB}$, which enabled accurate determination of false positives using the following formula: specificity = true negative number/(true negative number + false positive number) $\times$ $100 \%$. In this study, we compared the specificity of the developed ISET + combined fluorescent probe staining method with that of the ISET + Wright's Giemsa staining method by plotting the ROC curve and obtaining the AUC values. A higher AUC value of 0.844 was obtained for the combined fluorescent probe staining method compared to that of Wright's Giemsa staining (0.750). This indicates that the combined fluorescent probe staining method has higher CTC detection specificity than the Giemsa staining method.

\section{Correlation between CTC clinical detection accuracy of combined fluorescent probe staining method and clinicopathology}

We examined the correlation between CTC detection accuracy of the combined fluorescence staining method and clinicopathology of 32 tumor patients (22 males and 10 females), including 18 cases of esophageal cancer, six cases of liver cancer, four cases of renal cancer, one case of prostate cancer, one case of bladder cancer, one case of penile cancer, and one case of lung cancer. The 16 CTCpositive cases identified included seven cases of esophageal cancer, two cases of liver cancer, two cases of renal cancer, one case of prostate cancer, one case of bladder cancer, and one case of penile cancer. The 16 CTC-negative cases included 11 cases of esophageal cancer, four cases of liver cancer, two cases of renal cell carcinoma, and one case of penile cancer (Table 8). The CTC-positive rate was 11/22 for male patients and 5/10 for female patients (Table 16). CTCs were positively correlated with platelet levels (CTC-positive vs. CTC-negative: $261.71 \pm 42.21 \times 10^{9} / \mathrm{L} v s$. $211.73 \pm 71.20 \times 10^{9} / \mathrm{L} ; \mathrm{P}=0.031$ ) but were not associated with age, gender, drinking history, or granule ratio.

\section{Discussion}

In this study, we successfully verified the feasibility and established the protocol and CTC identification criteria for the novel combined fluorescent probe staining method. This method is an improvement to our previously established ISET-ICC CTC identification system. The combined fluorescent probe staining method showed consistent cell morphological characteristics (cell diameter, 
Table 7 Assessment of the performance of the novel combined fluorescent probe staining vs. Wright's Giemsa staining and CD45 ICC staining methods for in vivo CTC identification in 32 cancer patients

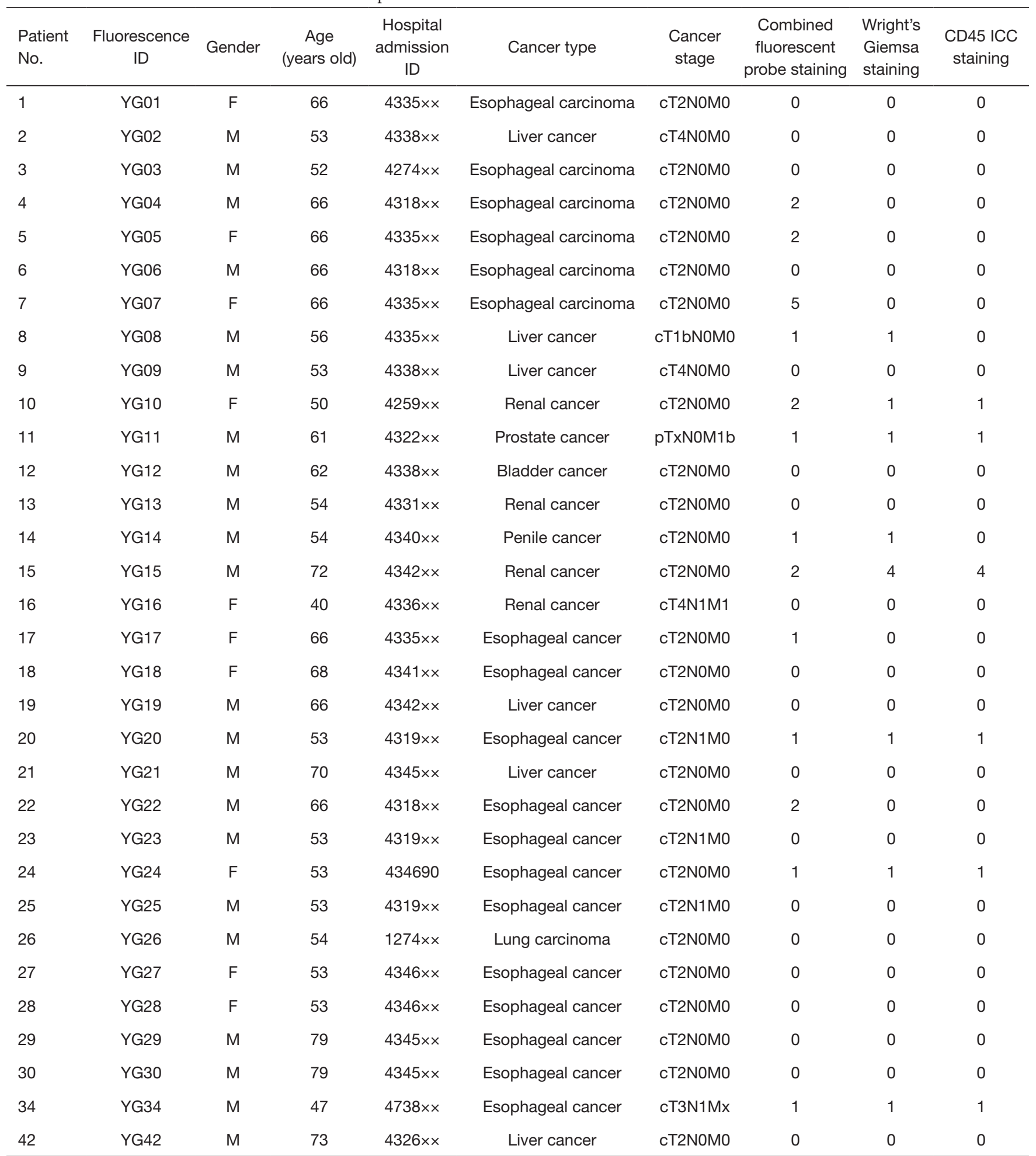

CD45, cluster differentiation 45; ICC, immunocytochemistry; CTC, circulating tumor cell; ID, Identification; F, female; M, male. 
Table 8 Comparison of the morphological characteristics of PB white blood cells in patient YG04 Li $\times$ Cheng among the three staining methods

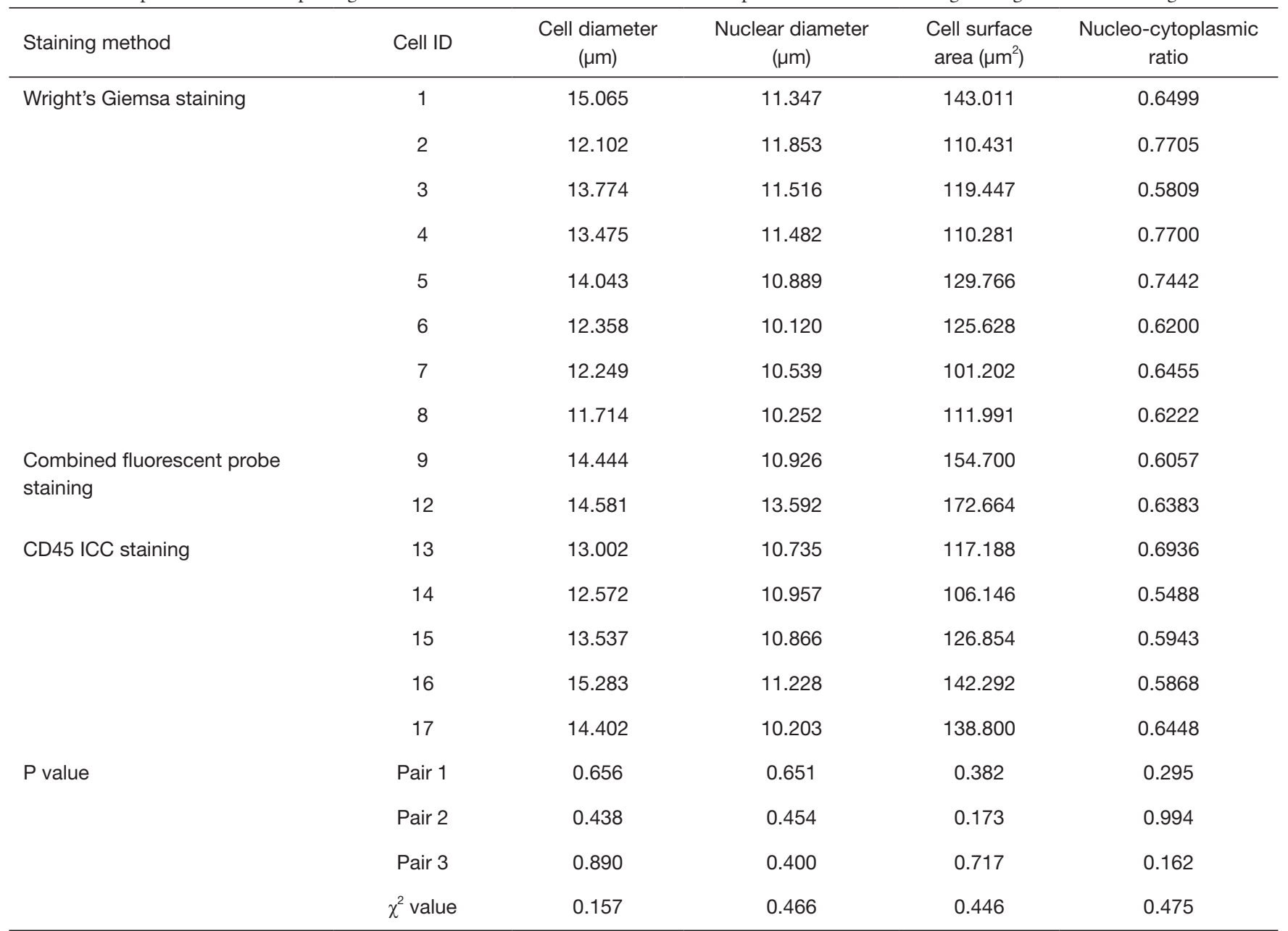

PB, peripheral blood; ID, identification; CD45, cluster differentiation 45; ICC, immunocytochemistry.

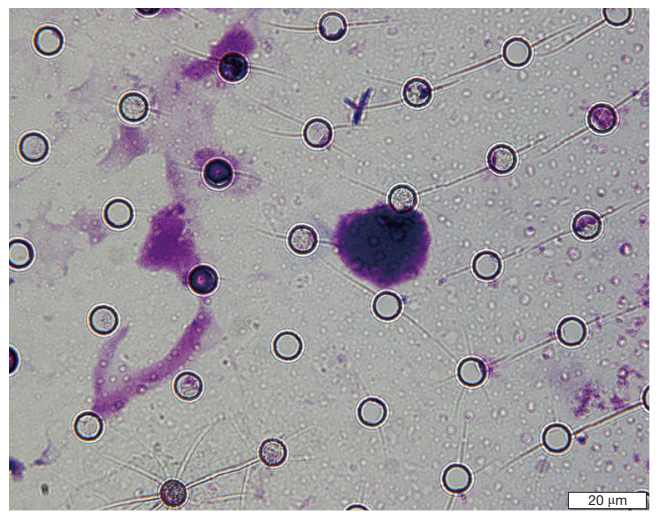

Figure 3 YG34 Chen $\times$ Wei (Giemsa staining; ×40).

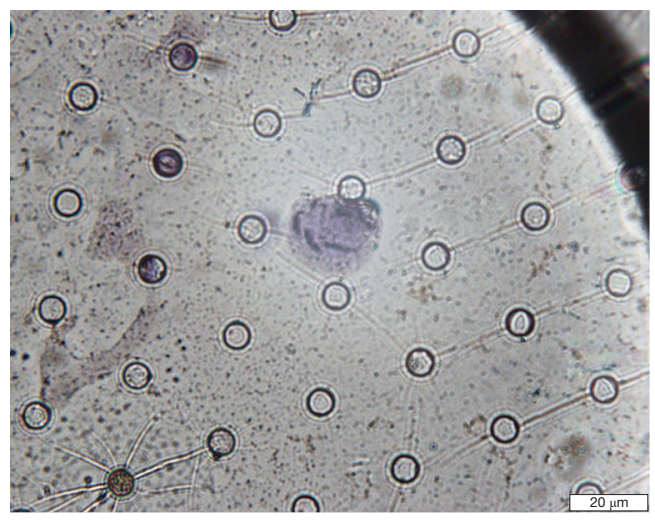

Figure 4 YG34 Chen $\times$ Wei (CD45 ICC staining; $\times 40)$. CD45, cluster differentiation 45 ; ICC, immunocytochemistry. 

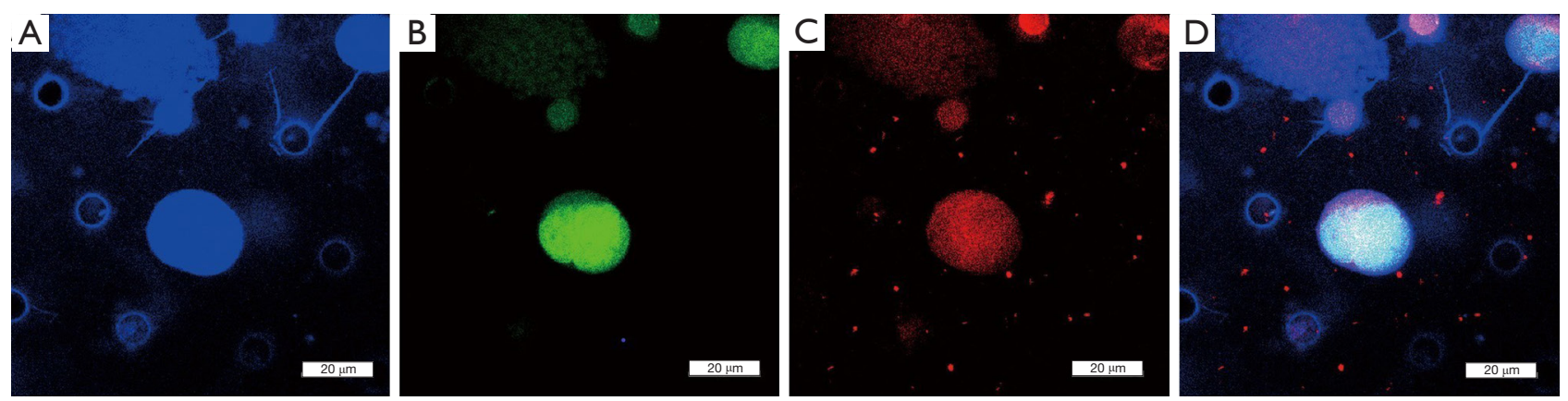

Figure 5 YG34 Chen $\times$ Wei [(A) blue marks the nucleu; (B) green marks the cytoplasmic; (C) red marks the cell membrane; (D) red and blue mark the fluorescent combination $\times 40)]$.

Table 9 Cell morphological characteristics of non-CTCs in PB detected by Wright's Giemsa staining

\begin{tabular}{|c|c|c|c|c|c|c|}
\hline Staining method & Slide ID & Cell ID & $\begin{array}{l}\text { Cell diameter } \\
(\mu \mathrm{m})\end{array}$ & $\begin{array}{c}\text { Nuclear } \\
\text { diameter }(\mu \mathrm{m})\end{array}$ & $\begin{array}{l}\text { Cell surface } \\
\text { area }\left(\mu m^{2}\right)\end{array}$ & $\begin{array}{c}\text { Nuclear: cytoplasmic } \\
\text { mass ratio }\end{array}$ \\
\hline \multirow[t]{6}{*}{ Wright's Giemsa staining } & 4 & 1 & 15.065 & 11.347 & 143.011 & 0.6499 \\
\hline & & 3 & 13.774 & 11.516 & 119.447 & 0.5809 \\
\hline & & 4 & 13.475 & 11.482 & 110.281 & 0.7700 \\
\hline & & 6 & 12.358 & 10.120 & 125.628 & 0.6200 \\
\hline & & 7 & 12.249 & 10.539 & 101.202 & 0.6455 \\
\hline & & 8 & 11.714 & 10.252 & 111.991 & 0.6222 \\
\hline
\end{tabular}

CTC, circulating tumor cell; PB, peripheral blood; ID, identification; SD, standard deviation.

Table 10 Cell morphological characteristics of non-CTCs in PB detected by combined fluorescent probe staining

\begin{tabular}{|c|c|c|c|c|c|c|}
\hline Staining method & Slide ID & Cell ID & $\begin{array}{l}\text { Cell diameter } \\
\qquad(\mu \mathrm{m})\end{array}$ & $\begin{array}{l}\text { Nuclear diameter } \\
(\mu \mathrm{m})\end{array}$ & $\begin{array}{l}\text { Cell surface } \\
\text { area }\left(\mu \mathrm{m}^{2}\right)\end{array}$ & $\begin{array}{l}\text { Nucleo-cytoplasmic } \\
\text { ratio }\end{array}$ \\
\hline \multirow{5}{*}{$\begin{array}{l}\text { Combined fluorescent } \\
\text { probe staining }\end{array}$} & 3 & 1 & 13.554 & 11.804 & 169.202 & 0.7145 \\
\hline & & 3 & 15.595 & 14.275 & 171.311 & 0.5898 \\
\hline & 4 & 9 & 14.444 & 10.926 & 154.700 & 0.6057 \\
\hline & 8 & 1 & 14.663 & 11.107 & 146.060 & 0.6516 \\
\hline & 9 & 1 & 15.949 & 11.385 & 206.625 & 0.5635 \\
\hline SD & & & 1.01652 & 0.34397 & 12.79116 & 0.06341 \\
\hline
\end{tabular}

CTC, circulating tumor cell; PB, peripheral blood; ID, identification; SD, standard deviation. 
Table 11 Cell morphological characteristics of non-CTCs in PB detected by CD45 ICC staining

\begin{tabular}{lcccccc}
\hline Staining method & Slide ID & Cell ID & Cell diameter $(\mu \mathrm{m})$ & Nuclear diameter $(\mu \mathrm{m})$ & Cell surface area $\left(\mu \mathrm{m}^{2}\right)$ & Nucleo-cytoplasmic ratio $^{2}$ \\
\hline CD45 ICC staining & 4 & 13 & 13.002 & 10.735 & 117.188 & 0.6936 \\
& 14 & 12.572 & 10.957 & 106.146 & 0.5488 & 0.5943 \\
& 15 & 13.537 & 126.854 & 142.292 & 0.5868 \\
& 16 & 15.283 & 11.228 & 138.800 & 0.6448 \\
SD & 17 & 14.402 & 0.62349 & 13.13872 & 0.07231 \\
Degree of skewness & & 1.12383 & 0.2865 & 0.3000 & 0.1234 \\
\hline
\end{tabular}

CTC, circulating tumor cell; PB, peripheral blood; CD45, cluster differentiation 45; ICC, immunocytochemistry; ID, identification; SD, standard deviation.

Table 12 Comparison of morphological characteristics of non-CTCs in PB among the three staining methods

\begin{tabular}{|c|c|c|c|c|c|c|}
\hline Staining method & Slide ID & Cell ID & Cell diameter $(\mu \mathrm{m})$ & Nuclear diameter $(\mu \mathrm{m})$ & Cell surface area $\left(\mu \mathrm{m}^{2}\right)$ & Nucleo-cytoplasmic ratio \\
\hline \multirow{6}{*}{$\begin{array}{l}\text { Wright's Giemsa } \\
\text { staining }\end{array}$} & \multirow{6}{*}{4} & 2 & 12.102 & 11.853 & 110.431 & 0.7705 \\
\hline & & 3 & 13.774 & 11.516 & 119.447 & 0.5809 \\
\hline & & 4 & 13.475 & 11.482 & 110.281 & 0.7700 \\
\hline & & 6 & 12.358 & 10.120 & 125.628 & 0.6200 \\
\hline & & 7 & 12.249 & 10.539 & 101.202 & 0.6455 \\
\hline & & 8 & 11.714 & 10.252 & 111.991 & 0.6222 \\
\hline \multirow{5}{*}{$\begin{array}{l}\text { Combined } \\
\text { fluorescent probe } \\
\text { staining }\end{array}$} & 3 & 1 & 13.554 & 11.804 & 169.202 & 0.7145 \\
\hline & \multirow[t]{2}{*}{4} & 9 & 14.444 & 10.926 & 154.700 & 0.6057 \\
\hline & & 12 & 14.581 & 13.592 & 172.664 & 0.6383 \\
\hline & 8 & 1 & 14.663 & 11.107 & 146.060 & 0.6516 \\
\hline & 9 & 1 & 15.949 & 11.385 & 206.625 & 0.5635 \\
\hline \multirow[t]{4}{*}{ CD45 ICC staining } & \multirow[t]{4}{*}{4} & 13 & 13.002 & 10.735 & 117.188 & 0.6936 \\
\hline & & 14 & 12.572 & 10.957 & 106.146 & 0.5488 \\
\hline & & 15 & 13.537 & 10.866 & 126.854 & 0.5943 \\
\hline & & 16 & 15.283 & 11.228 & 142.292 & 0.5868 \\
\hline
\end{tabular}

CTC, circulating tumor cell; PB, peripheral blood; ID, identification; CD45, cluster differentiation 45; ICC, immunocytochemistry. 
Table 13 Cell morphological characteristics of CTCs in PB that were subjected to Wright's Giemsa staining

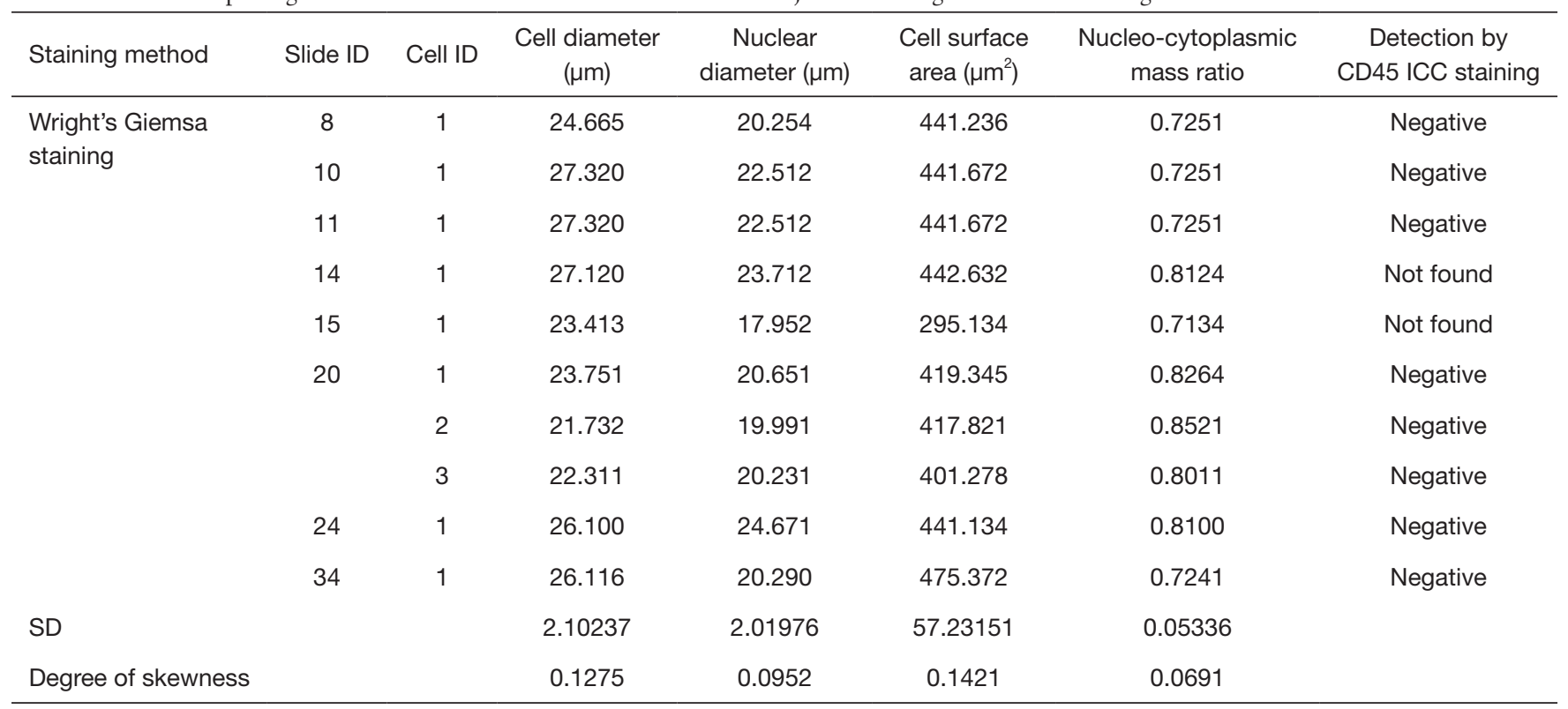

CTC, circulating tumor cell; PB, peripheral blood; ID, identification; CD45, cluster differentiation 45; ICC, immunocytochemistry; SD, standard deviation.

nuclear diameter, cell area, and nucleo-cytoplasmic ratio) for both CTCs and non-CTCs as Wright's Giemsa staining for in vitro CTC identification and similarly as Giemsa staining and CD45 ICC staining for in vivo CTC identification for the same samples examined. The newly developed combined fluorescent probe staining method exhibited higher CTC detection accuracy, as evident from the lower SD in the measurements for the cell morphological parameters examined and the higher AUC value compared to other reference control methods used. Investigation of the association between CTC detection by combined fluorescent probe staining and clinicopathology revealed a positive correlation with platelet count, but not in other assessed parameters. This suggests that platelet count may represent a potential biomarker for the presence of CTCs and tumor metastasis.

In the verification stage of the feasibility of the newly developed combined fluorescent probe staining method, the staining of cell morphological features in in vitro tumor cell lines by this method was compared to Giemsa staining. The absence of significant difference in the nucleocytoplasmic ratio in the five tumor cell lines between these two staining methods may be due to the following: (I) the lower fluorescence ratio of the combined fluorescent probe staining method used $(4,970)$ than the fluorescence intensities of cell $1(0.6320)$, cell $2(0.5653)$, and cell
$3(0.5945)$, as well as the average value of Wright's Giemsa staining (0.6726); and (II) only four cells in the combined fluorescent probe staining method met the measurement criteria, thus there was insufficient number of cells available for comparison.

Comparison between the combined fluorescent probe and Giemsa staining methods for the same 32 tumor samples revealed no significant differences in cell diameter and surface area, but significant differences in nuclear diameter and nucleo-cytoplasmic ratio. Moreover, the differences were more significant for the former method. This larger difference in CTC nuclear diameter and nucleocytoplasmic ratio may be due to the following: (I) variation in cell morphology and nuclear size; (II) both staining methods failed to detect CTCs; and (III) consecutive staining of the same sample with the combined fluorescent probe method followed by Giemsa staining may have affected CTC morphology. Collecting duplicate PB samples from the same tumor patients for in vivo experiments or performing the staining on two lots of the same tumor cell line for in vitro experiments instead of using the same samples for both combined fluorescent probe staining and Giemsa staining can clarify whether a significant difference exists. Another limitation that prevented a fair comparison between the two staining methods is the use of different microscopes for visualizing and imaging cell morphology; 
Table 14 Cell morphological characteristics of CTCs in PB that were subjected to combined fluorescent probe staining

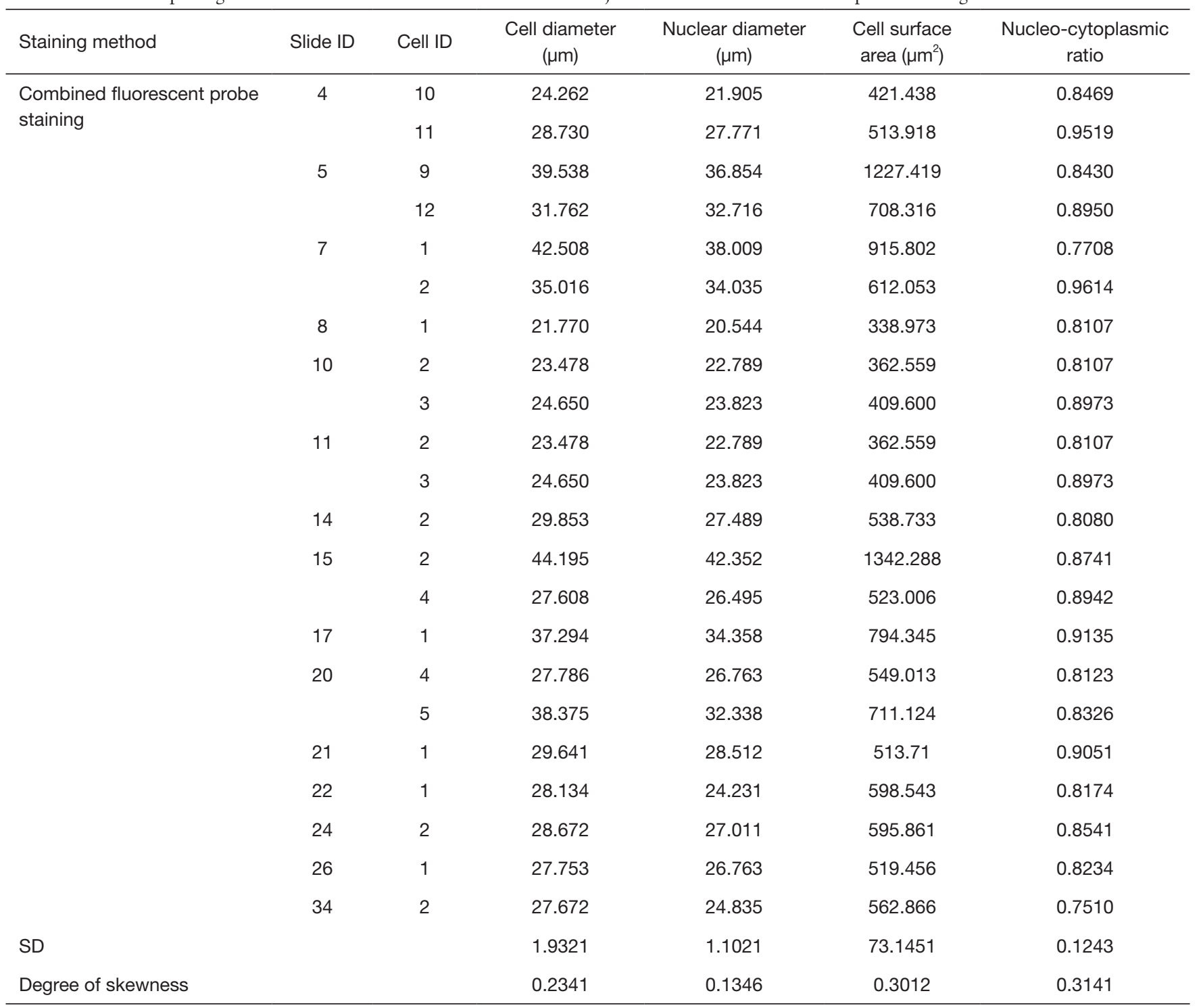

CTC, circulating tumor cell; PB, peripheral blood; ID, identification; SD, standard deviation.

a fluorescence microscope was used for cells stained with combined fluorescent probes, while a light microscope was used for Giemsa-stained cells.

As part of the comparison of the CTC detection rate and accuracy among the staining methods, we also compared the performance of these methods in identifying non-CTCs. Although we observed many non-CTCs in the random fields selected, we only selected three random cells for cell morphology measurements. Considering the large variation in measurement values of the assessed parameters for nonCTCs, we decided only to compare the morphological features of non-CTCs among the staining methods used.
There are few studies that have investigated the association between the CTC detection rate and cancer clinicopathology. In the present study, we found that CTC detection by combined fluorescent probe staining was positively correlated with platelet count. Our finding is consistent with two previous studies by our research group, which showed a correlation between platelet count and CTC detection rate in esophageal cancer $(1,2)$. Further research is necessary to better understand the implications and potential clinical application of the association between platelet count and CTC detection rate, as well as to determine whether this can be applied in tumor metastasis 
Table 15 Comparison of the cell morphological characteristics of CTCs in PB between Wright's Giemsa and combined fluorescent probe staining methods

\begin{tabular}{|c|c|c|c|c|c|c|}
\hline Staining method & Slide ID & Cell ID & $\begin{array}{l}\text { Cell diameter } \\
\qquad(\mu \mathrm{m})\end{array}$ & $\begin{array}{l}\text { Nuclear diameter } \\
\qquad(\mu \mathrm{m})\end{array}$ & $\begin{array}{l}\text { Cell surface area } \\
\qquad\left(\mu \mathrm{m}^{2}\right)\end{array}$ & $\begin{array}{c}\text { Nucleo-cytoplasmic } \\
\text { ratio }\end{array}$ \\
\hline \multirow{5}{*}{$\begin{array}{l}\text { Wright's Giemsa } \\
\text { staining }\end{array}$} & 8 & 1 & 24.665 & 20.254 & 441.236 & 0.7251 \\
\hline & 11 & 1 & 27.320 & 22.512 & 441.672 & 0.7251 \\
\hline & 20 & 1 & 23.751 & 20.651 & 419.345 & 0.8264 \\
\hline & & 2 & 21.732 & 19.991 & 417.821 & 0.8521 \\
\hline & & 3 & 22.311 & 20.231 & 401.278 & 0.8011 \\
\hline \multirow{16}{*}{$\begin{array}{l}\text { Combined fluorescent } \\
\text { probe staining }\end{array}$} & & 11 & 28.730 & 27.771 & 513.918 & 0.9519 \\
\hline & 5 & 9 & 39.538 & 36.854 & 1227.419 & 0.8430 \\
\hline & & 12 & 31.762 & 32.716 & 708.316 & 0.8950 \\
\hline & 7 & 1 & 42.508 & 38.009 & 915.802 & 0.7708 \\
\hline & & 2 & 35.016 & 34.035 & 612.053 & 0.9614 \\
\hline & 8 & 1 & 21.770 & 20.544 & 338.973 & 0.8107 \\
\hline & 10 & 2 & 23.478 & 22.789 & 362.559 & 0.8107 \\
\hline & & 4 & 27.608 & 26.495 & 523.006 & 0.8942 \\
\hline & 17 & 1 & 37.294 & 34.358 & 794.345 & 0.9135 \\
\hline & 20 & 4 & 27.786 & 26.763 & 549.013 & 0.8123 \\
\hline & & 5 & 38.375 & 32.338 & 711.124 & 0.8326 \\
\hline & 21 & 1 & 29.641 & 28.512 & 513.71 & 0.9051 \\
\hline & 22 & 1 & 28.134 & 24.231 & 598.543 & 0.8174 \\
\hline & 24 & 2 & 28.672 & 27.011 & 595.861 & 0.8541 \\
\hline & 26 & 1 & 27.753 & 26.763 & 519.456 & 0.8234 \\
\hline & 34 & 2 & 27.672 & 24.835 & 562.866 & 0.7510 \\
\hline$P$ value & & & 0.308 & 0.013 & 0.147 & 0.004 \\
\hline
\end{tabular}

CTC, circulating tumor cell; PB, peripheral blood; ID, identification. 
Table 16 Correlation between CTCs in PB detected by combined fluorescent probe staining and clinicopathological features of patients

\begin{tabular}{|c|c|c|c|c|}
\hline Parameters & Positive $(n=16)$ & Negative $(n=16)$ & Total & $P$ \\
\hline Gender & & & & $0.530^{\mathrm{a}}$ \\
\hline Male & 11 & 11 & 22 & \\
\hline Female & 5 & 5 & 10 & \\
\hline Platelet count & $261.71 \pm 42.21 \times 10$ & $211.73 \pm 71.20 \times 10^{b}$ & & $0.031^{\mathrm{b}}$ \\
\hline Granule/leaflet ratio & $2.8641 \pm 1.12$ & $2.2341 \pm 1.22$ & & $0.761^{\mathrm{b}}$ \\
\hline
\end{tabular}

${ }^{\mathrm{a}}, \chi^{2}$ test; ${ }^{\mathrm{b}}$, Student's $t$-test. CTC, circulating tumor cell; PB, peripheral blood.

prognosis. In addition, clinical validation of the newly developed combined fluorescent probe staining method with a larger patient cohort and stratifying patients based on cancer types can further provide insights into the true clinical applicability of this novel technique.

In this study, we successfully developed and validated the new combined fluorescent probe staining method for CTC identification. Notably, we demonstrated its higher CTC detection accuracy compared to the reference control, Giemsa staining method. Our study proposes the potential application of the novel staining method in clinical diagnostics and/or prognostics.

\section{Acknowledgments}

Funding: We thank the Ministry of Science and Technology Fund for the funding support (No. 2016YFC0106000).

\section{Footnote}

Reporting Checklist: The authors have completed the MDAR reporting checklist. Available at https://atm.amegroups. com/article/view/10.21037/atm-21-6476/rc

Data Sharing Statement: Available at https://atm.amegroups. com/article/view/10.21037/atm-21-6476/dss

Conflicts of Interest: All authors have completed the ICMJE uniform disclosure form (available at https://atm.amegroups. com/article/view/10.21037/atm-21-6476/coif). SH is from Wuhan YZY Medical Science and Technology Co. The other authors have no conflicts of interest to declare.

Ethical Statement: The authors are accountable for all aspects of the work in ensuring that questions related to the accuracy or integrity of any part of the work are appropriately investigated and resolved. All procedures performed in this study involving human participants were in accordance with the Declaration of Helsinki (as revised in 2013). The necessary approval was obtained from the Ethics Committee of Shandong Cancer Hospital and Institute, China (No. 201702019). Written consent was obtained from all eligible subjects before enrollment into the study.

Open Access Statement: This is an Open Access article distributed in accordance with the Creative Commons Attribution-NonCommercial-NoDerivs 4.0 International License (CC BY-NC-ND 4.0), which permits the noncommercial replication and distribution of the article with the strict proviso that no changes or edits are made and the original work is properly cited (including links to both the formal publication through the relevant DOI and the license). See: https://creativecommons.org/licenses/by-nc-nd/4.0/.

\section{References}

1. Yoon HJ, Kim TH, Zhang Z, et al. Sensitive capture of circulating tumour cells by functionalized graphene oxide nanosheets. Nat Nanotechnol 2013;8:735-41.

2. Hong B, Zu Y. Detecting circulating tumor cells: current challenges and new trends. Theranostics 2013;3:377-94.

3. Paget $\mathrm{S}$. The distribution of secondary growths in cancer of the breast. 1889. Cancer Metastasis Rev 1989;8:98-101.

4. Seiden MV, Kantoff PW, Krithivas K, et al. Detection of circulating tumor cells in men with localized prostate cancer. J Clin Oncol 1994;12:2634-9.

5. ENGELL HC. Cancer cells in the circulating blood; a clinical study on the occurrence of cancer cells in the 
peripheral blood and in venous blood draining the tumour area at operation. Acta Chir Scand Suppl 1955;201:1-70.

6. Jatana KR, Lang JC, Chalmers JJ. Identification of circulating tumor cells: a prognostic marker in squamous cell carcinoma of the head and neck? Future Oncol 2011;7:481-4.

7. Ankeny JS, Court CM, Hou S, et al. Circulating tumour cells as a biomarker for diagnosis and staging in pancreatic cancer. Br J Cancer 2016;114:1367-75.

8. Lorente D, Olmos D, Mateo J, et al. Circulating tumour cell increase as a biomarker of disease progression in metastatic castration-resistant prostate cancer patients with low baseline CTC counts. Ann Oncol 2018;29:1554-60.

9. Carles J, Castellano D, Méndez-Vidal MJ, et al. Circulating Tumor Cells as a Biomarker of Survival and Response to Radium-223 Therapy: Experience in a Cohort of Patients With Metastatic Castration-Resistant Prostate Cancer. Clin Genitourin Cancer 2018;16:e1133-9.

10. Terstappen LW, Rao C, Gross S, et al. Peripheral blood tumor cell load reflects the clinical activity of the disease in patients with carcinoma of the breast. Int J Oncol 2000;17:573-8.

11. Deng G, Herrler M, Burgess D, et al. Enrichment with anti-cytokeratin alone or combined with anti-EpCAM antibodies significantly increases the sensitivity for circulating tumor cell detection in metastatic breast cancer patients. Breast Cancer Res 2008;10:R69.

12. Liu Y, Zhang W, Sun Y, et al. Two-photon fluorescence imaging of RNA in nucleoli and cytoplasm in living cells based on low molecular weight probes. Dyes and Pigments 2014;103:191-201.

13. El-Heliebi A, Kroneis T, Zöhrer E, et al. Are morphological criteria sufficient for the identification of circulating tumor cells in renal cancer? J Transl Med 2013;11:214.

14. Song J, Nettles JB. Circulating tumor cells in patients with

Cite this article as: Wang H, Pei F, Li H, Li B, Han S, Yu $\mathrm{H}$, Li S. A novel combined fluorescent probe staining method for circulating tumor cell identification. Ann Transl Med 2022;10(2):50. doi: 10.21037/atm-21-6476 carcinoma in situ of the cervix uteri. Am J Obstet Gynecol 1969;104:713-26.

15. Hardingham JE, Kotasek D, Farmer B, et al. ImmunobeadPCR: a technique for the detection of circulating tumor cells using immunomagnetic beads and the polymerase chain reaction. Cancer Res 1993;53:3455-8.

16. Asworth TR. A case of cancer in which cells similar to those in tumors were seen in the blood after death. J Aust Med J 1869;14:146-9.

17. Vona G, Sabile A, Louha M, et al. Isolation by size of epithelial tumor cells : a new method for the immunomorphological and molecular characterization of circulatingtumor cells. Am J Pathol 2000;156:57-63.

18. Hofman V, Bonnetaud C, Ilie MI, et al. Preoperative circulating tumor cell detection using the isolation by size of epithelial tumor cell method for patients with lung cancer is a new prognostic biomarker. Clin Cancer Res 2011;17:827-35.

19. Gonzales PR, Mikhail FM. Diagnostic and Prognostic Utility of Fluorescence In situ Hybridization (FISH) Analysis in Acute Myeloid Leukemia. Curr Hematol Malig Rep 2017;12:568-73.

20. Frithiof H, Aaltonen K, Rydén L. A FISH-based method for assessment of HER-2 amplification status in breast cancer circulating tumor cells following CellSearch isolation. Onco Targets Ther 2016;9:7095-103.

21. Satelli A, Mitra A, Cutrera JJ, et al. Universal marker and detection tool for human sarcoma circulating tumor cells. Cancer Res 2014;74:1645-50.

22. Kolostova K, Spicka J, Matkowski R, et al. Isolation, primary culture, morphological and molecular characterization of circulating tumor cells in gynecological cancers. Am J Transl Res 2015;7:1203-13.

(English Language Editor: A. Kassem) 